Pacific Journal of Mathematics

THE LEVI PROBLEM FOR A PRODUCT MANIFOLD 


\section{THE LEVI PROBLEM FOR A PRODUCT MANIFOLD}

\section{Yasuo Matsugu}

Let $S$ be a Stein manifold, $T$ a one dimensional torus, $\pi$ a projection of the product $E=S \times T$ onto $S$ and $D$ a subdomain of $E$. The main object of this paper is to prove that $D$ is a Stein manifold if and only if $D$ is pseudoconvex in the sense of Cartan and $\pi^{-1}(x)$ is not contained in $D$ for any point $x$ of $S$.

1. A subdomain $D$ of a complex manifold $M$ is called pseudoconvex if, for any boundary point $x$ of $D$ in $M$, there is a Stein neighborhood $U$ of $x$ in $M$ such that $U \cap D$ is also a Stein manifold. A pair $(B, \beta)$ is called a domain over $M$ if $\beta$ is a locally biholomorphic mapping of a complex manifold $B$ in $M$. A domain $(B, \beta)$ over $C^{n}$ is called a domain of holomorphy if there exists a holomorphic function $f$ in $B$ such that the radius of convergence of $f$ at any point $x$ of $B$ is just the boundary distance $d(x)$ of $x$.

Moreover we recall another definition. Let $\varphi_{i}: M_{i} \rightarrow N_{i}$ be two mappings of a set $M_{i}$ into a set $N_{i}(i=1,2)$. Then we define the product mapping $\varphi_{1} \times \varphi_{2}$ of the product set $M_{1} \times M_{2}$ into the product set $N_{1} \times N_{2}$ by putting $\left(\varphi_{1} \times \varphi_{2}\right)(x, y)=\left(\varphi_{1}(x), \varphi_{2}(y)\right)$ for $(x, y) \in M_{1} \times M_{2}$.

The proof of our theorem falls into two parts. We first prove it in the case of $S=C^{n}$, where we construct a strongly plurisubharmonic function by means of Hörmander [2] and reduce it to a result of Narasimhan [3]. In general case, using the imbedding of Docquier-Grauert [1], we reduce the theorem to the case of $C^{n}$.

2. Let $(B, \beta)$ be a domain of holomorphy over $C^{n}$. In the complex plane $C$ select any two complex numbers $\omega_{1}, \omega_{2}$ which are linearly independent over the real number field $R$. The numbers $\omega_{1}, \omega_{2}$ generate a subgroup $\Gamma$ of $C$, namely

$$
\Gamma=\left\{m_{1} \omega_{1}+m_{2} \omega_{2} ; m_{1}, m_{2} Z=\text { addtive group of integers }\right\} \text {. }
$$

The quotient $T=C / \Gamma$ is a one dimensional torus. $T$ has a natural complex structure and is a compact Riemann surface. The natural map $\tau: C \rightarrow T$ is a locally biholomorphic map. We denote by $E=$ $B \times T$ the product of two complex manifolds $B$ and $T$, and by $\pi: E \rightarrow B$ the projection.

We first prove the following lemma:

Lemma. Let $D$ be a pseudoconvex open subset of $E$ such that $\pi^{-1}(x)$ is not contained in $D$ for any point $x$ of $B$. Then $D$ is a Stein manifold. 
Proof. Let $1 \times \tau$ be the product map of the identity 1 of $B$ and the map $\tau$. The map $1 \times \tau$ is a locally biholomorphic map $B \times C$ onto $E$. If we denote by $A$ the inverse image $(1 \times \tau)^{-1}(D)$ of $D, A$ is pseudoconvex, because $D$ is pseudoconvex. $A$ is $\Gamma$-invariant, that is, for any fixed point $\gamma \in \Gamma, A$ is invariant under the transformation of $B \times C:(y, z) \mapsto(y, z+\gamma)$. Let $\alpha$ be the restriction to $A$ of the product map $\beta \times 1$ of the map $\beta$ and the identity map 1 of $C$, that is, $\alpha(y, z)=(\beta(y), z)$ for $(y, z) \in A$. $\alpha$ is a locally biholomorphic map of $A$ into $C^{n} \times C=C^{n+1}$ and $(A, \alpha)$ is a pseudoconvex domain over $C^{n+1}$. The distance function $d(y, z)$ of the domain $A$ over $C^{n+1}$ induces the function $d(y, t)$ in $D$. Indeed, for any point $(y, t) \in D, y \in B, t \in T$, select two representatives $z, z^{\prime} \in C$ of the equivalence class $t$. Then there is $\gamma \in \Gamma$ such that $z^{\prime}=z+\gamma$. But $A$ is $\Gamma$-invariant, and so $d\left(y, z^{\prime}\right)=d(y, z)$. Since $A$ is pseudoconvex, by Oka [4], the function $-\log d(y, z)$ is a continuous plurisubharmonic function in $A$. The function $-\log d(y, t)$ is therefore a continuous plurisubharmonic function in $D$, and so is the function

$$
1 / d(y, t)=e^{-\log d(y, t)} .
$$

On the other hand, since $B$ is Stein, there is a real analytic strongly plurisubharmonic function $q>0$ with the following property: for any real number $c>0$,

$$
B_{c}=\{y \in B ; q(y)<c\} \subseteq B .
$$

The function

$$
\gamma(y, t)=\frac{1}{d(y, t)}+q(y)
$$

defined in $D$ is a continuous plurisubharmonic function. It holds that

$$
\begin{aligned}
D_{c}= & \{(y, t) \in D ; \gamma(y, t)<c\} \\
& \subset B_{c} \times T \cap\left\{(y, t) \in D ; d(y, t)>\frac{1}{c}\right\} \Subset D
\end{aligned}
$$

for any real number $c>0$.

Since $D=\bigcup_{c>0} D_{c}$, if we show that $D_{c}$ is a Stein manifold, we know by Docquier-Grauert [1], that $D$ is itself a Stein manifold.

Fix an arbitrary real number $c>0$. For any point $y \in B$, we set

$$
A(y)=\{z \in C ;(y, \tau(z)) \in D\} .
$$

By the hypothesis of the lemma, it follows that $A(y) \varsubsetneqq C$. Select a complex-valued measurable function $a(y)$ in $B$ such that

$$
a(y) \in C-A(y) \text { for any point } y \in B .
$$


For sufficiently small number $\varepsilon$ with $0<\varepsilon<1 /(c+1)<1 / c$, we define the function $s(y, t)$ in $D_{c+1}$ as follows:

$$
s(y, t)=\frac{1}{\varepsilon^{2 n}} \int_{\xi \in B} \rho\left(\frac{y-\xi}{\varepsilon}\right) \sum_{m_{1}, m_{2}=-\infty}^{+\infty} \frac{d \lambda(\xi)}{\left|z-a(\xi)-m_{1} \omega_{1}-m_{2} \omega_{2}\right|^{2}},
$$

where $\rho$ is Friedrichs' modifier, and $z$ in the summation $\sum$ is a representative of $t$. Clearly the sum $\Sigma$ converges uniformly, and does not depend on any choice of representative $z$.

Moreover, we define a function $p(y, t)$ in $D_{c+1}$ by putting

$$
p(y, t)=s(y, t)+K q(y)
$$

where $K$ is a sufficient large constant. Since $D_{c} \Subset D_{c+1}$ and $q$ is a strongly plurisubharmonic function in $B$, it follows that the function $p(y, t)$ is strongly plurisubharmonic in $D_{c}$. By Narasimhan [3], we can conclude that $D_{c}$ is a Stein manifold.

3. Now we shall prove our main theorem.

Theorem. Let $E$ be the product $S \times T$ of a Stein manifold $S$ and a complex torus $T$, and $\pi$ be the projection $E \rightarrow S$. Let $D$ be an open subset of $E$. Then $D$ is a Stein manifold if and only if $D$ is pseudoconvex and $\pi^{-1}(x)$ is not contained in $D$ for any point $x \in S$.

Proof. By Docquier-Grauert [1], there are a biholomorphic map $\sigma$ of $S$ onto a regular analytic set of a domain of holomorphy $(B, \beta)$ over $C^{n}$ and a holomorphic mapping $\rho$ of $B$ onto $\sigma(S)$ such that the restriction $\rho \mid \sigma(S)$ is the identiny of $\sigma(S)$. We define a mapping $\xi$ of the product $G=B \times T$ onto $E=S \times T$ by putting $\xi(x, t)=$ $\left(\sigma^{-1}(\rho(x)), t\right)$ for $(x, t) \in G$. The inverse image $\xi^{-1}(D)$ of $D$ under the map is a pseudoconvex open subset of $G$ and satisfies the hypothesis of the lemma. $\xi^{-1}(D)$ is therefore a Stein manifold. Since $D$ is a regular analytic subset of the Stein manifold $\xi^{-1}(D), D$ is also a Stein manifold.

\section{REFERENCES}

1. F. Docquier und H. Grauert, Levisches Problem und Rungerscher Satz für Teilgebiete Steinscher Mannigfaltigkeiten, Math. Ann., 140 (1960), 94-123.

2. L. Hörmander, An Introduction to Complex Analysis in Several Variables, D. Van. Nostrand (1966).

3. R. Narasimhan, The Levi problem for complex spaces II, Math. Ann., 146 (1962), 195-216.

4. K. Oka, Sur les fonctions de plusieurs variables. IX. Domaines finis sans point critique interieur, Japan J. Math., 23 (1953), 97-155.

Received February 1, 1972 and in revised November 13, 1972.

KYUShU UNIVERSITy 



\section{PACIFIC JOURNAL OF MATHEMATICS}

\section{EDITORS}

D. Gilbarg and J. Milgram Stanford University

Stanford, California 94305

R. A. Beaumont

University of Washington

Seattle, Washington 98105
J. DugundJI

Department of Mathematics

University of Southern California

Los Angeles, California 90007

RICHARD ARENS

University of California

Los Angeles, California 90024

ASSOCIATE EDITORS
E. F. BECKENBACH
B. H. NeumanN
F. WOLF
K. YoSHIDA

\section{SUPPORTING INSTITUTIONS}

UNIVERSITY OF BRITISH COLUMBIA

CALIFORNIA INSTITUTE OF TECHNOLOGY

UNIVERSITY OF CALIFORNIA

MONTANA STATE UNIVERSITY

UNIVERSITY OF NEVADA

NEW MEXICO STATE UNIVERSITY

OREGON STATE UNIVERSITY

UNIVERSITY OF OREGON

OSAKA UNIVERSITY
UNIVERSITY OF SOUTHERN CALIFORNIA

STANFORD UNIVERSITY

UNIVERSITY OF TOKYO

UNIVERSITY OF UTAH

WASHINGTON STATE UNIVERSITY

UNIVERSITY OF WASHINGTON

$\stackrel{*}{*} \stackrel{*}{*} \stackrel{*}{*}$ AMERICAN MATHEMATICAL SOCIETY

NAVAL WEAPONS CENTER 


\section{Pacific Journal of Mathematics}

\section{Vol. 46, No. $1 \quad$ November, 1973}

Allan Francis Abrahamse, Uniform integrability of derivatives on $\sigma$-lattices .......................................... 1

Ronald Alter and K. K. Kubota, The diophantine equation $x^{2}+D=p^{n} \ldots \ldots \quad 11$

Grahame Bennett, Some inclusion theorems for sequence spaces .......... 17

William Cutler, On extending isotopies ........................ 31

Robert Jay Daverman, Factored codimension one cells in Euclidean

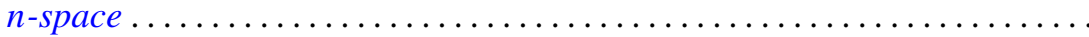

Patrick Barry Eberlein and Barrett O’Neill, Visibility manifolds ............ 45

M. Edelstein, Concerning dentability .......................... 111

Edward Graham Evans, Jr., Krull-Schmidt and cancellation over local

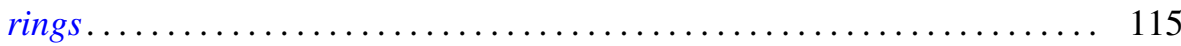

C. D. Feustel, A generalization of Kneser's conjecture ................ 123

Avner Friedman, Uniqueness for the Cauchy problem for degenerate parabolic equations .......................................... 131

David Golber, The cohomological description of a torus action ............ 149

Alain Goullet de Rugy, Un théorème du genre "Andô-Edwards" pour les

Fréchet ordonnés normaux..............................

Louise Hay, The class of recursively enumerable subsets of a recursively enumerable set ........................................

John Paul Helm, Albert Ronald da Silva Meyer and Paul Ruel Young, On orders of translations and enumerations...

Julien O. Hennefeld, A decomposition for $B(X)^{*}$ and unique Hahn-Banach

extensions

Gordon G. Johnson, Moment sequences in Hilbert space .

Thomas Rollin Kramer, A note on countably subparacompact spaces ...

Yves A. Lequain, Differential simplicity and extensions of a derivation ....

Peter Lorimer, A property of the groups Aut $\mathrm{PU}\left(3, q^{2}\right) \ldots$

225

Yasou Matsugu, The Levi problem for a product manifold.

231

John M.F. O'Connell, Real parts of uniform algebras ......

William Lindall Paschke, A factorable Banach algebra without bounded

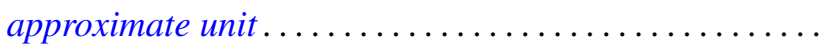

Ronald Joel Rudman, On the fundamental unit of a purely cubic field ....

Tsuan Wu Ting, Torsional rigidities in the elastic-plastic torsion of simply connected cylindrical bars .........................

Philip C. Tonne, Matrix representations for linear transformations on analytic sequences...................................

Jung-Hsien Tsai, On E-compact spaces and generalizations of perfect

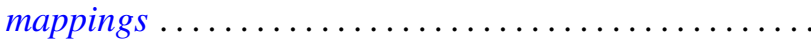

Alfons Van Daele, The upper envelope of invariant functionals majorized by an invariant weight. .. 